\title{
Article \\ Geodesic Mappings of Semi-Riemannian Manifolds with a Degenerate Metric
}

\author{
Igor G. Shandra ${ }^{1}\left(\mathbb{D}\right.$ and Josef Mikeš ${ }^{2, *(\mathbb{D}}$ \\ 1 Department of Mathematics, Financial University under the Goverment the Russian Federation, \\ 125468 Moscow, Russia; ma-tematika@yandex.ru \\ 2 Department of Algebra and Geometry, Faculty of Science, Palacky University, \\ 77146 Olomouc, Czech Republic \\ * Correspondence: josef.mikes@upol.cz
}

Citation: Shandra, I.G.; Mikeš, J.

Geodesic Mappings of Semi-

Riemannian Manifolds with a

Degenerate Metric. Mathematics 2022,

10, 154. https://doi.org/10.3390/

math10010154

Academic Editor: Sorin V. Sabau

Received: 14 December 2021

Accepted: 31 December 2021

Published: 5 January 2022

Publisher's Note: MDPI stays neutral with regard to jurisdictional claims in published maps and institutional affiliations.

Copyright: () 2022 by the authors Licensee MDPI, Basel, Switzerland. This article is an open access article distributed under the terms and conditions of the Creative Commons Attribution (CC BY) license (https:// creativecommons.org/licenses/by/ $4.0 /)$.

\begin{abstract}
This article introduces the concept of geodesic mappings of manifolds with idempotent pseudo-connections. The basic equations of canonical geodesic mappings of manifolds with completely idempotent pseudo-connectivity and semi-Riemannian manifolds with a degenerate metric are obtained. It is proved that semi-Riemannian manifolds admitting concircular fields admit completely canonical geodesic mappings and form a closed class with respect to these mappings.
\end{abstract}

Keywords: semi-Riemannian manifold; degenerate metric; pseudo-connection; concircular vector field; geodesic mapping

MSC: 53C20; 53C21; 53C24

\section{Introduction}

The problem of geodesic mappings of Riemannian manifolds was first introduced by T. Levi-Civita in the study of problems in mechanics [1]. There are many monographs and papers devoted to the theory of geodesic mappings and transformations, their generalizations, and applications [2-20]. In addition, A. Z. Petrov [11] used geodesic mappings and their generalizations of pseudo-Riemannian spaces for models of gravitation fields. The above-mentioned spaces that generalize semi-Riemannian spaces with degenerate metrics are found in various applications, in particular unified field theories. As it was shown in [14], in the case when the torsion tensor is semisymmetric, setting the Levi-Civita pseudo-connection is equivalent to setting the Weyl connection used in a unified field theory combining gravity and electromagnetism. Linear idempotent operators are used to define calibration fields that define different types of interactions. The theory of the multidimensional Universe uses degenerate Kaluza-Klein metrics [21,22].

The basic equations of geodesic mappings for pseudo-Riemannian manifolds were obtained by Levi-Civita, but they were non-linear $[8,14,16,18]$. The basic equations of geodesic mappings for pseudo-Riemannian manifolds in linear form were obtained by N. S. Sinyukov [16]. These equations greatly advanced the study of geodesic maps and allowed us to obtain many interesting results. In particular, it reduced the question of whether a given pseudo-Riemannian manifold admits a non-trivial geodesic mapping to the analysis of a system of linear algebraic equations.

Analogues of the Sinyukov equations for holomorphic-projective mappings of Kähler manifolds were obtained by J. Mikes [8]. However, all existing generalizations of geodesic mappings assume that the metric tensor of a pseudo-Riemannian manifold is nondegenerate. However, in physics and mechanics, there are models in which the metric tensor is degenerate [15].

In this paper, we generalize the results of geodesic mappings of pseudo-Riemannian manifolds to the case of semi-Riemannian spaces with a degenerate metric. In particular, 
we will obtain analogues of the Levi-Civita equations and the Sinyukov equations. For our research, we use the theory of idempotent pseudo-connections [15].

\section{Preliminaries}

Let $M_{n}$ be a smooth $n$-dimensional manifold. We denote the ring of smooth functions on $M_{n}$ by $C^{\infty}\left(M_{n}\right)$, the Lie algebra of smooth vector fields on $M_{n}$ by $\chi\left(M_{n}\right)$, and arbitrary smooth vector fields on $M_{n}$ by $X, Y, Z$, and $W$.

Definition 1. A linear pseudo-connection on $M_{n}$ is a pair of operators $(h ; \nabla)$, where $\nabla: \chi\left(M_{n}\right) \times$ $\chi\left(M_{n}\right) \rightarrow \chi\left(M_{n}\right)$ and $h$ is a linear operator on $\chi\left(M_{n}\right)$, which for $X, Y, Z \in \chi\left(M_{n}\right), f \in C^{\infty}\left(M_{n}\right)$ satisfies the following conditions [14]:

$$
\begin{gathered}
\nabla_{X}(f Y+Z)=f \nabla_{X} Y+X(f) \cdot h Y+\nabla_{X} Z \\
\nabla_{f X+Y} Z=f \nabla_{X} Z+\nabla_{Y} Z .
\end{gathered}
$$

In the case where $h=\mathrm{id}$, any linear pseudo-connection is a linear connection on $M_{n}$.

Definition 2. The torsion and curvature tensors of the linear pseudo-connection $(h ; \nabla)$ are defined as follows [14]:

$$
S(X, Y)=\nabla_{X} Y-\nabla_{Y} X-h[X, Y] \text { and } R(X, Y) Z=\nabla_{X} \nabla_{Y} Z-\nabla_{Y} \nabla_{X} Z-\nabla_{[X, Y]} Z \text {. }
$$

Definition 3. A linear pseudo-connection $(h ; \nabla)$ is said to be idempotent if it satisfies the following conditions [14]:

$$
\begin{aligned}
& h^{2}=h ; \\
& \nabla=h \nabla .
\end{aligned}
$$

In this case, $h$ is called the horizontal projector, and $v=\mathrm{id}-h$ is called the vertical projector. Here, $\nabla=h \nabla$ means $\nabla_{X} Y=h \nabla_{X} Y$.

The torsion and curvature tensors of an idempotent pseudo-connection satisfy the following conditions [14]:

$$
\begin{gathered}
v S(X, Y)=0 \\
v R(X, Y) Z=0
\end{gathered}
$$

Definition 4. A linear pseudo-connection $(h ; \nabla)$ is said to be completely idempotent if it satisfies the following conditions [14]:

$$
h^{2}=h \text { and } \nabla=h \nabla h,
$$

where $\nabla=h \nabla h$ means $\nabla_{X} Y=h \nabla_{X}(h Y)$.

A manifold on which is given a completely idempotent pseudo-connection $(h ; \nabla)$ with Rank $h=r$ is denoted by $A_{h}^{r}$. The completely idempotent pseudo-connection is an idempotent pseudo-connection [14].

The torsion and curvature tensors of a completely idempotent pseudo-connection satisfy the following conditions [14]:

$$
\begin{gathered}
S(v X, v Y)=-h[v X, v Y] ; \\
v R(X, Y) Z=R(X, Y) v Z=0 .
\end{gathered}
$$

Definition 5. A pair $(h ; g)$, where $h$ is a linear operator and $g$ is a bilinear form, is called an $H R$-structure of rank $r$ if they satisfy the following conditions [14]:

$$
h^{2}=h
$$




$$
\begin{gathered}
g(h X, Y)=g(X, Y)=g(Y, X) ; \\
\text { Rank } h=\text { Rank } g=r \leq n .
\end{gathered}
$$

A manifold $M_{n}$ with an HR-structure is called a semi-Riemannian manifold and is denoted by $V_{n}^{r}$

For any $H R$-structure $(h ; g)$, there is a unique linear pseudo-connection $(h ; \nabla)$, called the Levi-Civita pseudo-connection, that satisfies the conditions [14]

$$
\begin{gathered}
\nabla g=0 ; \\
g(S(X, h Y), Z)=g(S(X, h Z), Y) .
\end{gathered}
$$

It is defined by the formula [14]

$$
\begin{array}{r}
2 g\left(\nabla_{X} Y, Z\right)=X g(Y, Z)+(h Y) g(X, Z)-(h Z) g(X, Y) \\
+g(h Y, X, Z)+g([h Z, X], Y)-g(X,[h Z, h Y]) .
\end{array}
$$

\section{Geodesic Mappings of Manifolds with an Idempotent Pseudo-Connection}

Let $M_{n}$ be an $n$-dimensional manifold with an idempotent pseudo-connection $(h ; \nabla)$.

Definition 6. A curve $\tau(t)$ on $M_{n}$ is called a geodesic if it satisfies the following condition:

$$
\nabla_{X} X=\gamma h X,
$$

where $X$ is a tangent vector of $\tau$, and $\gamma$ is a function of parameter $t$.

Let $h_{j}^{i}, \Gamma_{j k}^{i}$ be components of the pseudo-connection $(h ; \nabla)$, and $X^{i}$ be components of the tangent vector $X$ in some coordinate system on $M_{n}$. Then, Equation (10) can be written in the equivalent form

$$
h_{k}^{i} \frac{d X^{k}}{d t}+\Gamma_{j k}^{i} X^{j} X^{k}=\gamma h_{k}^{i} X^{k}
$$

We remark that a curve $\tau(t)$ on $M_{n}$ with an affine connection $\tilde{\nabla}$ is called an F-planar curve if it satisfies [8]

$$
\tilde{\nabla}_{X} X=\alpha X+\beta F X,
$$

where $F$ is a linear operator, and $\alpha$ and $\beta$ are some functions of $t$.

If $F$ is an almost product structure $\left(F^{2}=\mathrm{id}\right)$, then

$$
h=\frac{F+\mathrm{id}}{2} \text { and } v=\frac{F-\mathrm{id}}{2}
$$

are horizontal and vertical projectors, respectively. Then,

$$
h \tilde{\nabla}_{X} X=(\alpha+\beta) h X \text { and } v \tilde{\nabla}_{X} X=(\alpha-\beta) v X .
$$

It follows from (9) that the curve $\tau$ is the geodesic curve with respect to the pseudoconnection $(h ; h \tilde{\nabla})$ and from (10) that the curve $\tilde{\tau}$ is the geodesic with respect to the pseudo-connection $(v ; v \tilde{\nabla})$.

Definition 7. A diffeomorphism $f: M_{n} \rightarrow \bar{M}_{n}$ is called a geodesic mapping of $M_{n}$ onto $\bar{M}_{n}$ if $f$ maps any geodesic on $M_{n}$ onto a geodesic on $\bar{M}_{n}$.

Theorem 1. A manifold $M_{n}$ with an idempotent pseudo-connection $(h, \nabla)$ admits a geodesic mapping onto a manifold $\bar{M}_{n}$ with the idempotent pseudo-connection $(h, \bar{\nabla})$ if and only if the equation

$$
\bar{\nabla}_{X} Y=\nabla_{X} Y+\psi(X) h Y+\psi(Y) h X+N(X, Y)
$$


holds for any vector fields $X, Y$, where $\psi$ is a differential form on $M_{n}\left(=\bar{M}_{n}\right)$, and the $N(X, Y)$ tensor satisfies the following conditions:

$$
\begin{gathered}
N(X, Y)=N(Y, X) \\
h N(X, Y)=N(X, Y) .
\end{gathered}
$$

Proof. Let $f: M_{n} \rightarrow \bar{M}_{n}$ be a geodesic mapping. Therefore, a geodesic $\tau$ on $M_{n}$ maps onto a geodesic $\bar{\tau}$ on $\bar{M}_{n}$. Then, in a common coordinate system $\left(x^{i}\right)$ with respect the mapping $f$, the curve $\tau$ satisfies (11), and $\bar{\tau}$ satisfies the following conditions:

$$
h_{k}^{i} \frac{d X^{k}}{d t}+\bar{\Gamma}_{j k}^{i} X^{j} X^{k}=\bar{\gamma} h_{k}^{i} X^{k} .
$$

Subtracting Equation (11) from this equation, we obtain

$$
\left(\bar{\Gamma}_{j k}^{i}-\Gamma_{j k}^{i}\right) X^{j} X^{k}=(\bar{\gamma}-\gamma) h_{k}^{i} X^{k} .
$$

Multiplying the above formula by $h_{m}^{l} X^{m}$, and alternating by $i$ and $l$, we obtain

$$
\left(P_{j k}^{i} h_{m}^{l}-P_{j k}^{l} h_{m}^{i}\right) X^{j} X^{k} X^{m}=0,
$$

where

$$
P_{j k}^{i}=\left(\bar{\Gamma}_{j k}^{i}+\bar{\Gamma}_{k j}^{i}-\Gamma_{j k}^{i}-\Gamma_{k j}^{i}\right) .
$$

The relations (15) are fulfilled identically with respect to $X$, so it follows from (15) that

$$
P_{j k}^{i} h_{m}^{l}+P_{m j}^{i} h_{k}^{l}+P_{k m}^{i} h_{j}^{l}-P_{j k}^{l} h_{m}^{i}-P_{m j}^{l} h_{k}^{i}-P_{k m}^{l} h_{j}^{i}=0 .
$$

Due to (2)

$$
P_{j k}^{i}=P_{j k}^{m} h_{m}^{i} .
$$

Thus, contracting (16) in $j$ and $m$, we obtain

$$
P_{j k}^{i}(r-1)+P_{m j}^{i} h_{j}^{m}+P_{k m}^{i} h_{k}^{m}-P_{m j}^{m} h_{k}^{i}-P_{k m}^{m} h_{j}^{i}=0 .
$$

It follows from (17) that

$$
P_{j k}^{i}=\psi_{j} h_{k}^{i}+\psi_{k} h_{j}^{i}=0,
$$

where

$$
\psi_{j}=\frac{1}{r} P_{m s}^{m} v_{j}^{s}+\frac{1}{r+1} P_{m s}^{m} h_{j}^{s} \quad \text { or } \quad P_{j k}^{i}=\psi_{j} h_{k}^{i}+\psi_{k} h_{j}^{i} .
$$

Thus, we have found the symmetric part of the deformation tensor

$$
T_{j k}^{i}=\bar{\Gamma}_{j k}^{i}-\Gamma_{j k}^{i} .
$$

Thus,

$$
T_{j k}^{i}=\psi_{j} h_{k}^{i}+\psi_{k} h_{j}^{i}+N_{j k^{\prime}}^{i}
$$

where

$$
N_{j k}^{i}=\bar{S}_{j k}^{i}-S_{j k}^{i} .
$$

Thus,

$$
N_{j k}^{i}=-N_{k j}^{i}
$$

and due to (3),

$$
N_{j k}^{i}=N_{k j}^{m} h_{m}^{i} .
$$

The conditions (18)-(20) are equivalent to (12)-(14). Conversely, it is easy to check that if the conditions (12)-(14) hold, then any geodesic on $M_{n}$ will be a geodesic on $\bar{M}_{n}$. 
Theorem 2. Let

$$
S(h X, h Y)=\bar{S}(h X, h Y) .
$$

Then, a manifold $A_{n}^{r}$ with a completely idempotent pseudo-connection $(h, \nabla)$ admits a geodesic mapping onto a manifold $\bar{A}_{n}^{r}$ with a completely idempotent pseudo-connection $(h, \bar{\nabla})$ if and only if the equation

$$
\bar{\nabla}_{X} Y=\nabla_{X} Y+\psi(h X) h Y+\psi(h Y) h X+2 \psi(v X) h Y
$$

holds for any vector fields $X, Y$, where $\psi$ is a differential form on $A_{n}^{r}\left(=\bar{A}_{n}^{r}\right)$.

Proof. We have from (12)

$$
\bar{\nabla}_{X}(v Y)=\nabla_{X}(v Y)+\psi(v Y) h X+N(X, v Y) .
$$

Taking into account (5), we obtain

$$
\bar{\nabla}_{X}(v Y)=\nabla_{X}(v Y)=0
$$

Thus, we have from (23) and (24)

$$
N(X, v Y)=-\psi(v Y) h X
$$

It follows from (25) that

$$
\begin{gathered}
N(v X, v Y)=0 \\
N(h X, v Y)=-\psi(v Y) h X .
\end{gathered}
$$

In addition, according to (21), we obtain

$$
N(h X, h Y)=0 .
$$

We have

$$
N(X, Y)=N(v X, v Y)+N(v X, h Y)+N(h X, v Y)+N(h X, h Y)
$$

We obtain from (29), due to (26)-(28),

$$
N(X, Y)=\psi(v Y) h X+\psi(v X) h Y
$$

Substituting (30) into (12), we find

$$
\bar{\nabla}_{X} Y=\nabla_{X} Y+\psi(X) h Y+\psi(Y) h X-\psi(v Y) h X+\psi(v X) h Y
$$

or

$$
\bar{\nabla}_{X} Y=\nabla_{X} Y+\psi(h X) h Y+\psi(h Y) h X+2 \psi(v X) h Y .
$$

The theorem is proved.

Definition 8. If $\psi=0$, then geodesic mapping is called trivial, and nontrivial if $\psi \neq 0$.

Definition 9. A geodesic mapping of a manifold $A_{n}^{r}$ with a completely idempotent pseudoconnection $(h, \nabla)$ onto a manifold $\bar{A}_{n}^{r}$ with a completely idempotent pseudo-connection $(\bar{h}, \bar{\nabla})$ is called canonical if

$$
\begin{gathered}
h=\bar{h} \\
S(X, Y)=\bar{S}(X, Y) .
\end{gathered}
$$


Corollary 1. A manifold $A_{n}^{r}$ with a completely idempotent pseudo-connection $(h, \nabla)$ admits a canonical geodesic mapping onto a manifold $\bar{A}_{n}^{r}$ with a completely idempotent pseudo-connection $(h, \bar{\nabla})$ if and only if in the Equation (22),

$$
\psi(v X)=0 .
$$

Proof. The condition (32) is equivalent to $N(X, Y)=0$. Thus, if $N(X, Y)=0$, then we have from (25) that $\psi(v X)=0$. Conversely, if $\psi(v X)=0$, then we obtain from (30) that $N(X, Y)=0$. The corollary is proved.

It follows from (22) that the equation of a canonical geodesic mapping of manifolds with a completely idempotent pseudo-connection due to (33) is equivalent to the equation

$$
\begin{gathered}
\bar{\nabla}_{X} Y=\nabla_{X} Y+\psi(X) h Y+\psi(Y) h X ; \\
\psi(h X)=\psi(X) .
\end{gathered}
$$

The Equations (34) and (35) can be rewritten in the coordinate form as

$$
\begin{gathered}
\bar{\Gamma}_{j k}^{i}=\Gamma_{j k}^{i}+\psi_{j} \delta_{k}^{i}+\psi_{k} \delta_{j}^{i} ; \\
\psi_{i} h_{k}^{i}=\psi_{k},
\end{gathered}
$$

and these equations are the generalization of the equations of geodesic mappings of manifolds with an affine connection $[8,16]$.

\section{Completely Canonical Geodesic Mappings of Semi-Riemannian Manifolds}

Let $V_{n}^{r}=\left(M_{n}, g, h\right)$ be a semi-Riemannian manifold with an $H R$-structure $(h, r)$ and $\nabla$ be a Levi-Civita pseudo-connection.

Theorem 3. A semi-Riemannian manifold $V_{n}^{r}=\left(M_{n}, g, h\right)$ admits a canonical geodesic mapping onto a semi-Riemannian manifold $\bar{V}_{n}^{r}=\left(\bar{M}_{n}, \bar{g}, h\right)$ if and only if there exists a differential form $\psi(X)$ on $V_{n}^{r}$ such that equations

$$
\begin{gathered}
\left(\nabla_{Z \bar{g}}\right)(X, Y)=2 \psi(Z) \bar{g}(X, Y)+\psi(X) \bar{g}(Y, Z)+\psi(Y) \bar{g}(X, Z) \\
\psi(h X)=\psi(X) \\
\bar{g}(S(X, h Y), Z)=\bar{g}(S(X, h Z), Y)
\end{gathered}
$$

hold for any vector fields $X, Y, Z$.

The validity of this statement follows from (8), (32), (34), and (35).

The coordinate form of Equations (38) and (39) can be given by the following formulas:

$$
\begin{gathered}
\nabla_{k} \bar{g}_{i j}=2 \psi_{k} \bar{g}_{i j}+\psi_{i} \bar{g}_{j k}+\psi_{j} \bar{g}_{j k} ; \\
\bar{g}_{k l} S_{i m}^{l} h_{j}^{m}=\bar{g}_{j l} S_{i m}^{l} h_{k}^{m} .
\end{gathered}
$$

Equations (35), (38), and (39) are the generalization of the equations of geodesic mappings of pseudo-Riemannian manifolds [8,16].

Definition 10. A canonical geodesic mapping of a semi-Riemannian manifold $V_{n}^{r}$ onto a semiRiemannian manifold $\bar{V}_{n}^{r}$ is called completely canonical if there exists a function $\Psi$ such that in Equation (38) satisfies $\psi=d \Psi$, and in the coordinate form

$$
\psi_{i}=\partial_{i} \Psi
$$

This shows that $\psi$ is a gradient covector. 
Theorem 4. If the affinor $h$ of the HR-structure $(h, g)$ is integrable then any canonical geodesic mapping of a semi-Riemannian manifold $V_{n}^{r}$ is completely canonical.

Proof. If the affinor $h$ of the $H R$-structure $(h, g)$ is integrable, then there exists the adapted coordinate system $x^{i}=\left(x^{I}, x^{\alpha}\right)$ on $V_{n}^{r}$ that the components of $h$ reduce to the form

$$
h_{j}^{i}=\left(\begin{array}{cc}
\delta_{J}^{I} & 0 \\
0 & 0
\end{array}\right)
$$

where $I, J$ follow from 1 to $r$, and $\alpha, \beta$ follow from $r+1$ to $n$. It follows from (6), (7), and (43) that in this coordinate system,

$$
g_{i j}=\left(\begin{array}{cc}
G_{I J} & 0 \\
0 & 0
\end{array}\right) \text { and } g^{i j}=\left(\begin{array}{cc}
G^{I J} & 0 \\
0 & 0
\end{array}\right) \text {, }
$$

where $g^{i j}$ is the semi-inverse matrix to $g_{i j}$; thus,

$$
g^{i k} g_{k j}=h_{j}^{i}
$$

and $G^{I J}$ is the inverse matrix to $G_{I J}$

$$
\operatorname{det}\left(G_{I J}\right)=r
$$

Contracting (36) in $i$ and $j$, we obtain $\psi_{k}(r+1)=\bar{\Gamma}_{i k}^{i}-\Gamma_{i k}^{i}$. It follows from (9) and (44)-(46) that

$$
\psi_{k}(r+1)=\frac{1}{2} \partial_{k} \bar{G}_{I J} \bar{G}^{I J}-\frac{1}{2} \partial_{k} G_{I J} G^{I J},
$$

or

$$
\psi_{k}(r+1)=\frac{1}{2}\left(\partial_{k}(\ln |\bar{G}|)-\partial_{k}(\ln |G|)\right)=\frac{1}{2} \partial_{k}\left(\frac{\ln |\bar{G}|}{\ln |G|}\right) .
$$

The theorem is proved.

Theorem 5. A semi-Riemannian manifold $V_{n}^{r}$ admits a completely canonical geodesic mapping if and only if there exist a differential form $\lambda(X)$ and a bilinear form $a(X, Y)$ on $V_{n}$ such that the equations

$$
\begin{gathered}
\left(\nabla_{Z} a\right)(X, Y)=\lambda(X) g(Y, Z)+\lambda(Y) g(X, Z) ; \\
a(X, Y)=a(Y, X)=a(h X, Y) ; \\
\operatorname{Rank} a=r ; \\
a(S(X, h Y), Z)=a(S(X, h Z), Y) ; \\
\lambda(h X)=\lambda(X)
\end{gathered}
$$

hold for any vector fields $X, Y, Z$.

Proof. Let $\bar{g}^{i j}$ be the components of a semi-inverse tensor to $\bar{g}_{i j}$; thus,

$$
\bar{g}^{i l} \bar{g}_{l j}=h_{j}^{i}
$$

Then, it follows from (40) and (41) by virtue of (52) that

$$
\begin{gathered}
\nabla_{k} \bar{g}^{i j}=2 \psi_{k} \bar{g}^{i j}+\psi^{i} h_{k}^{j}+\psi^{j} h_{k}^{i} ; \\
\bar{g}^{m k} S_{i m}^{l} h_{l}^{j}=\bar{g}^{m j} S_{i m}^{l} h_{l}^{k},
\end{gathered}
$$

where $\psi^{i}=\bar{g}^{i l} \psi_{l}$. 
Let us denote

$$
a_{i j}=\exp (2 \Psi) \bar{g}^{s t} g_{s i} g_{t j} .
$$

It easy to find from (54), due to (53), the following equations:

$$
\begin{gathered}
\nabla_{k} a_{i j}=\lambda_{i} g_{j k}+\lambda_{j} g_{i k} ; \\
a_{i j}=a_{j i}=a_{i l} h_{j}^{l} \\
\operatorname{Rank}\left(a_{i j}\right)=r ; \\
a_{i l} S_{m k}^{l} h_{j}^{m}=a_{j l} S_{m k}^{l} h_{i}^{m} ; \\
\lambda_{l} h_{i}^{l}=\lambda_{i},
\end{gathered}
$$

where

$$
\lambda_{i}=-\exp (2 \Psi) \bar{g}^{s l} g_{s i} \psi_{l} .
$$

Equations (55)-(59) are equivalent to (47)-(51).

Conversely, if there exist a differential form $\lambda(X)$ and a bilinear form $a(X, Y)$ on $V_{n}$ such that Equations (55)-(59) hold, then there exist an $H R$-structure $(h, g)$ and a differential form $\phi(X)$ such that Equations (37)-(39) and (42) hold, where

$$
\begin{gathered}
\psi_{i}=-\lambda_{s} a^{s t} g_{t i} ; \\
\bar{g}_{i j}=\exp (2 \Psi) a^{s t} g_{s i} g_{t j} .
\end{gathered}
$$

The theorem is proved.

Contracting (55) by $g^{i j}$ in $i$ and $j$, we obtain $2 \lambda_{i}=\partial_{i}\left(a_{s t} g^{s t}\right)$.Thus, $\lambda_{i}$ is a gradient covector.

It follows from (60) and (61) that $\psi \neq 0$ if and only if $\lambda \neq 0$.

Equations (47)-(51) generalize N. S. Sinyukov's equations for geodesic mappings of pseudo-Riemannian manifolds $[8,16]$.

Equation (55) can be rewritten in the equivalent form

$$
\begin{gathered}
h_{k}^{l} \nabla_{l} a_{i j}=\lambda_{i} g_{j k}+\lambda_{j} g_{i k} ; \\
v_{k}^{l} \nabla_{l} a_{i j}=0 .
\end{gathered}
$$

The integrability conditions of Equation (55) on the basis of (62), (63), and the Ricci identities take the following form [14]:

$$
\begin{gathered}
a_{s(i} R_{j) t m}^{s} h_{k}^{t} h_{l}^{m}=g_{i[l} h_{k]}^{t} \nabla_{t} \lambda_{j}+g_{j[l} h_{k]}^{t} \nabla_{t} \lambda_{i} ; \\
a_{s(i} R_{j) t m}^{s} v_{k}^{t} v_{l}^{m}=v_{k}^{t} v_{l}^{m} S_{l m}^{s} g_{s(i} \lambda_{j)} ; \\
a_{s(i} R_{j) t m}^{s} h_{k}^{t} v_{l}^{m}=h_{k}^{t} v_{l}^{m} S_{l m}^{s} g_{s(i} \lambda_{j)}+v_{k}^{t} \nabla_{t} \lambda_{\left(i g_{j) k}\right.},
\end{gathered}
$$

where $R_{i k l}^{j}$ are components of the curvature tensor $R$ :

$$
R\left(\partial_{k}, \partial_{l}\right) \partial_{l}=R_{i k l}^{j} \partial_{j}
$$

Contracting (64) by $g^{j k}$ in $j$ and $k$, and (66) by $g^{i j}$ in $i$ and $j$, we find

$$
\begin{gathered}
r h_{l}^{t} \nabla_{t} \lambda_{i}=\mu g_{i j}+h_{l}^{m}\left(a_{s t} R_{i}{ }^{s t}{ }_{m}+a_{s t} R^{s t}{ }_{t m}\right) ; \\
v_{l}^{t} \nabla_{t} \lambda_{i}=h_{i}^{t} v_{l}^{m} S_{i m}^{s} \lambda_{s} .
\end{gathered}
$$


Thus, we obtain from (67) and (68)

$$
\nabla_{l} \lambda_{i}=\frac{1}{r}\left(\mu g_{i j}+h_{l}^{m}\left(a_{s t} R_{i}^{s t}{ }_{m}+a_{s t} R^{s t}{ }_{t m}\right)\right)+h_{i}^{t} v_{l}^{m} S_{t m}^{s} \lambda_{s}
$$

where $\mu$ is a certain scalar field.

Similarly, analysing the integrability conditions of Equation (69) on the basis of (67) and (68), it is not difficult to obtain equations that $\mu$ satisfies:

$$
\begin{aligned}
\nabla_{k} \mu= & \frac{a_{s t}}{1-r}\left(2 h_{k}^{m} \nabla_{m} R^{s t}-\nabla^{t} R_{k}^{s}-\nabla^{m} R_{m}^{s} k^{r}\right)+R_{m}^{t} v_{k}^{m} \lambda_{t}-\frac{2 \mu}{r} S_{k m}^{t} v_{k}^{m} \\
& +\frac{\lambda_{t}}{1-r}\left(2(r+1) R_{k}^{t}+r H_{k m}^{s} S^{m t}{ }_{s}\right)+\frac{2 a_{s t} v_{k}^{l}}{r}\left(R^{t m} h_{m}^{p} S_{p l}^{s}-R^{s}{ }_{m p}^{t} h_{q}^{p} S^{m q}{ }_{l}\right),
\end{aligned}
$$

where $R_{i k}=R_{i k t}^{t}$ is the Ricci tensor, and $H_{j k}^{t}$ is the nonholonomy tensor of the horizontal distribution $H(X, Y)=v[h X, h Y]$.

Thus, the following theorem is proved.

Theorem 6. In order that a semi-Riemannian manifold $V_{n}^{r}$ admit a completely canonical geodesic mapping, it is necessary and sufficient that the system (55)-(59), (69), and (70) has a solution $\left(a_{i j}, \lambda_{i}, \mu\right)$.

Theorem 6 is a generalization of the main theorem of geodesic mappings of pseudoRiemannian manifolds. The system of Equations (55), (69), and (70) forms a closed system of first-order linear partial differential equations of the Cauchy type. The integrability conditions of these equations, as well as their differential prolongations, will also be linear. Thus, the question of whether a given semi-Riemannian manifold $V_{n}^{r}$ admits a completely canonical geodesic mapping is reduced to the analysis of the consistency of a certain system of linear algebraic equations.

\section{Completely Canonical Geodesic Mappings and Concircular Fields}

Definition 11. A vector field $\varphi$ on a semi-Riemannian manifold $V_{n}^{r}$ satisfying the conditions [13]

$$
\begin{gathered}
\left(\nabla_{Z} \varphi\right) X=\varrho g(X, Z) ; \\
\varphi(h X)=\varphi(X),
\end{gathered}
$$

where $\varrho$ is a scalar field on $V_{n}^{r}$, is called a concircular field on $V_{n}^{r}$. Here, we mean the covariant derivative of the covector field. A covariant derivative with respect to the pseudo-connection can be defined for a tensor field of any type. You can read about this in [14].

The Equations (71) and (72) can be rewritten in the equivalent coordinate form

$$
\begin{gathered}
\nabla_{i} \varphi_{j}=\varrho g_{i j} \\
\varphi_{t} h_{i}^{t}=\varphi_{i} .
\end{gathered}
$$

If $\varrho \neq 0$, a concircular field belongs to the main type, and it belongs to the exceptional type otherwise.

Theorem 7. Let $\varphi$ be a concircular field on a semi-Riemannian manifold $V_{n}^{r}$. If $V_{n}^{r}=\left(M_{n}, g, h\right)$ admits a nontrivial completely canonical geodesic mapping onto a semi-Riemannian manifold $\bar{V}_{n}^{r}=\left(\bar{M}_{n}, \bar{g}, h\right)$, then there exists a concircular field $\bar{\varphi}$ on $\bar{V}_{n}^{r}$.

Proof. Let $\varphi$ be the components of the concircular field $\varphi$ on $V_{n}^{r}$. Then,

$$
\bar{\varphi}_{i}=\exp (-\Psi) \varphi_{s} \bar{g}^{s t} g_{t i}
$$


is the components of the concircular field $\bar{\varphi}$ on $\bar{V}_{n}^{r}$ due to (36) and

$$
\bar{\varrho}=\exp (-\Psi)\left(\varrho+\psi_{s} \varphi^{s}\right)
$$

The theorem is proved.

Theorem 8. Let $\varphi$ be a concircular field of the main type on a semi-Riemannian manifold $V_{n}^{r}$; then, $V_{n}^{r}$ admits a nontrivial completely canonical geodesic mapping.

Proof. Let $\varphi_{i}$ be the components of the concircular field $\varphi$ on $V_{n}^{r}$. Then, the tensor

$$
a_{i j}=\varphi_{i} \varphi_{j}+C g_{i j}
$$

satisfies (55)-(57) and (59), where the constant $C$ is chosen in a way that $\operatorname{Rank}\left(a_{i j}\right)=r$ and where

$$
\lambda_{i}=\varrho \varphi_{i} .
$$

It follows from the integrability conditions of Equation (73) that

$$
\varphi_{t} S_{i m}^{t} v_{j}^{m}=v_{j}^{m} \partial_{m}(\ln |\varrho|) \varphi_{i} .
$$

We have, due to (74),

$$
\varphi_{j} \varphi_{t} S_{i m}^{t} v_{k}^{m}=v_{k}^{m} \partial_{m}(\ln |\varrho|) \varphi_{j} \varphi_{i}=\varphi_{i} \varphi_{t} S_{j m}^{t} v_{k}^{m} .
$$

Whereas, for the Levi-Civita pseudo-connection

$$
S_{m t}^{k} h_{i}^{m} h_{j}^{t}=0
$$

it follows from (75) and (76) that tensor $a_{i j}$ satisfies (58). Thus, $a_{i j}$ satisfies (55)-(59), and according to Theorem 5 , the space $V_{n}^{r}$ admits a nontrivial completely canonical geodesic mapping. The theorem is proved.

\section{Conclusions}

In this paper, we study geodesic mappings of manifolds with idempotent pseudoconnections. We obtained the basic equations of canonical geodesic mappings of manifolds with completely idempotent pseudo-connectivity and semi-Riemannian manifolds with a degenerate metric. We proved that semi-Riemannian manifolds admitting concircular fields admit completely canonical geodesic mappings and form a closed class with respect to these mappings.

Author Contributions: I.G.S. and J.M. contributed equally and significantly in writing this article. All authors have read and agreed to the published version of the manuscript.

Funding: This research was funded by the grant IGA PrF 2021030 at Palacky University in Olomouc for the second author only.

Conflicts of Interest: The authors declare no conflict of interest.

\section{References}

1. Levi-Civita, T. Sulle transformationi dello equazioni dinamiche. Ann. Mat. Milano 1886, 24, 255-300.

2. Aminova, A.V. Projective transformations of pseudo-Riemannian manifolds. J. Math. Sci. N. Y. 2003, 113, 367-470. [CrossRef]

3. Berezovski, V.; Cherevko, Y.; Hinterleitner, I.; Peška, P. Geodesic mappings of spaces with affine connections onto generalized symmetric and Ricci-symmetric spaces. Mathematics 2020, 8, 1560. [CrossRef]

4. Berezovski, V.; Mikeš, J.; Radulović, Ž. Almost geodesic mappings of type $\pi_{1}^{*}$ of spaces with almost geodesic mappings of type of spaces with affine connection. Math. Montisnigro 2021, 52, 30-36. [CrossRef]

5. Ćirić, M.S.; Zlatanović, M.L.; Stanković, M.S.; Velimirović, L.S. On geodesic mappings of equidistant generalized Riemannian spaces. Appl. Math. Comput. 2012, 218, 6648-6655. [CrossRef]

6. Eisenhart, L.P. Riemannian Geometry; Princeton University Press: Princeton, NJ, USA, 1949. 
7. Hall, G. Projective structure in space-times. AMS/IP Stud. Adv. Math. 2011, 49, 71-79.

8. Mikeš, J. et al. Differential Geometry of Special Mappings; Palacky University Press: Olomouc, Czech Republic, 2015.

9. Mikeš, J.; Hinterleitner, I.; Guseva, N.I. Geodesic maps "in the large" of Ricci-flat spaces with $n$ complete geodesic lines. Math. Notes 2020, 108, 292-296. [CrossRef]

10. Norden, A.P. Spaces of Affine Connection; Nauka: Moscow, Russia, 1976.

11. Petrov, A.Z. New Methods in the General Theory of Relativity; Nauka: Moscow, Russia, 1966.

12. Petrović, M.Z.; Velimirović, A.M. Projective curvature tensors of some special manifolds with non-symmetric linear connection. Mediterr. J. Math. 2021, 18, 1-16. [CrossRef]

13. Shandra, I.G. On concircular tensor fields and geodesic mappings of pseudo-Riemannian spaces. Russ. Math. $2001,45,52-62$.

14. Shandra, I.G. Pseudoconnections and manifolds with degenerate metrics. J. Math. Sci. 2004, 119, 658-681. [CrossRef]

15. Shandra, I.G.; Mikeš, J. Geodesic mappings of V(K)-space. Mathematics 2019, 7, 692. [CrossRef]

16. Sinyukov, N.S. Geodesic Mappings of Riemannian Spaces; Nauka: Moscow, Russia, 1979.

17. Stanković, M.S.; Minčić, S.M.; Velimirović, L.S.; Zlatanović, M.L. On equitorsion geodesic mappings of general affine connection spaces. Rend. Semin. Mat. Univ. Padova 2010, 124, 77-90. [CrossRef]

18. Stepanov, S.E.; Shandra, I.G.; Mikeš, J. Harmonic and projective diffeomorphisms. J. Math. Sci. 2015, 207, 658-668. [CrossRef]

19. Yildirim, G.C.; Arsan, G.G. Geodesic mappings between Kähler-Weyl spaces. Differ. Geom. Dyn. Syst. 2007, 9, 156-159.

20. Zlatanović, M.L.; Velimirović, L.S.; Stanković, M.S. Necessary and sufficient conditions for equitorsion geodesic mapping. J. Math. Anal. Appl. 2016, 435, 578-592. [CrossRef]

21. Coquereaux, P.; Jadczyk, A. Geometry of multidimential Universe. Comm. Math. Phys. 1983, 90, 79-100. [CrossRef]

22. Ivanenko, D.D.; Sardnashvili, G.A. Gravitation; Naukova Dumka: Kiev, Ukraine, 1985. 